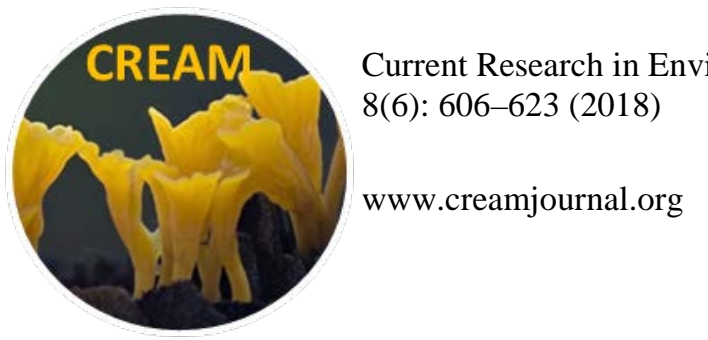

ISSN 2229-2225

\title{
A preliminary study on domestication of wild-growing medicinal mushrooms collected from Northern Iran
}

\author{
Shahtahmasebi SH ${ }^{1}$, Pourianfar HR $^{1 *}$, Mahdizadeh $\mathbf{V}^{2}$, Shahzadeh Fazeli SA ${ }^{3,4}$, \\ Amoozegar $\mathrm{MA}^{2,5}$, Nasr $\mathrm{SH}^{2}$, Zabihi $\mathrm{SS}^{2}$, Rezaeian $\mathrm{SH}^{1}$, Malekzadeh $\mathrm{KH}^{6}$, \\ Janpoor $\mathbf{J}^{1}$
}

${ }^{1}$ Industrial Fungi Biotechnology Research Department, Research Institute for Industrial Biotechnology, Academic
Center for Education, Culture and Research (ACECR)- Khorasan Razavi, P.O.Box: 91775-1376, Mashhad, Iran
${ }^{2}$ Microorganisms Bank, Iranian Biological Resource Center (IBRC), Academic Center for Education, Culture and
Research (ACECR)-Tehran, Iran
${ }^{3}$ Human and Animal Cell Bank, Iranian Biological Resource Center (IBRC), Academic Center for Education, Culture
and Research (ACECR)-Tehran, Iran
${ }^{4}$ Department of Molecular and Cellular Biology, Faculty of Basic Sciences and Advanced Technologies in Biology,
University of Science and Culture, Tehran, Iran
${ }^{5}$ Extremophiles Laboratory, Department of Microbiology, Faculty of Biology and Center of Excellence in Phylogeny of
Living Organisms, College of Science, University of Tehran, Iran
${ }^{6}$ Faculty of Agriculture, Vali-e-Asr University of Rafsanjan, Kerman, Iran

Shahtahmasebi SH, Pourianfar HR, Mahdizadeh V, Shahzadeh Fazeli SA, Amoozegar MA, Nasr SH, Zabihi SS, Rezaeian SH, Malekzadeh KH, Janpoor J 2018 - A preliminary study on domestication of wild-growing medicinal mushrooms collected from Northern Iran. Current Research in Environmental \& Applied Mycology (Journal of Fungal Biology) 8(6), 606-623, Doi 10.5943/cream/8/6/4

\begin{abstract}
Domestication of wild mushrooms and preserving their mycelia alive is essential to ensure reproducibility of biomedical research and breeding programs. This study was conducted with the aim of domestication of wild-growing mushrooms collected from the northern Iran (Mazandaran province) where is known to be rich in valuable higher fungal reserves. Totally, 40 well-identified wild isolates belonging to Basidiomycota (comprised of 21 different genera) was evaluated in solid media (potato dextrose agar and compost extract agar), spawn (grain- and wood-based spawn), and lignocellulosic substrate (wood chips-based substrate supplemented with 15\% wheat bran). Significant differences were found between the isolates and between the media in terms of mycelial growth $(p<0.05)$. While more than half of the isolates showed a growth rate higher than $3 \mathrm{~mm}$ per day, few isolates exhibited a growth rate of less than one mm per day in both media. Besides, two isolates failed to grow in grain-based or wood-based spawn, while most of them colonized wheat grains shorter than wood chips $(p<0.05)$. The fruiting tests showed that eight isolates were able to produce fruiting bodies in the substrate, including: Pholiota aurivella (GPS 142), Lenzites tricolor (GPS 180), Ganoderma tsugae (GPS 186), Cyclocybe sp. (Darabkola 20), Trametes sp. (GPS 130), Trametes sp. (Darabkola 13), Daedaleopsis sp. (Royan 8), and Donkia pulcherrima (Neka 24D). Based on the literature survey, most of these isolates possess antimicrobial and anticancer properties. Therefore, adaptation of these wild species to produce fruiting bodies may facilitate further research on their biological activities.
\end{abstract}


Key words - Adaptation - Basidiomycota - Fruiting bodies - Mycelial growth - Wild mushrooms

\section{Introduction}

The local knowledge about production and medicinal properties of non-Agaricus wild mushrooms (known as specialty mushrooms) in the East and Southeast Asia dates back to centuries ago (Oyetayo 2011, Wachtel-Galor et al. 2011). However, only less than 10 mushroom species out of 14,000 taxonomically known wild mushroom species around the world have reached large-scale commercial cultivation (Miles \& Chang 2004). Among these commercially cultivated species, five main genera of mushrooms, including Agaricus, Lentinula, Pleurotus, Auricularia, and Flammulina account for 85\% of the global mushroom production (Royse et al. 2017).

Despite the global trend in cultivation of non-Agaricus specialty mushrooms, the white button mushroom (Agaricus bisporus) is still the most cultivated species in countries such as Iran, where is known to be rich in a large number of different species of wild mushrooms (Rahnama \& Habibi 2015). A vast number of studies have reported the presence of wild mushrooms in different provinces of the country (Ershad 2009, Asef 2010). Among these regions, forests located in the north of Iran have temperate climate, adequate vegetation, and sufficient moisture, making them suitable to host a wide range of different types of edible, medicinal, industrial, and symbiotic macrofungi. Thus, a significant number of studies performed on Iranian wild-growing macrofungi are related to Mazandaran as one of the Northern provinces. Examples published in English journals include (but not limited to) collection of 100 species of macroscopic fungi from Mazandaran, of which 11 species were recorded as new species in Iran (Borhani et al. 2010). A study on Ganoderma spp. also reported collection of several Ganoderma spp. from Northern Iran (Keypour et al. 2014). A more recent study collected more than 470 specimens from Kheyroud Educational and Experimental Forest (Mazandaran), of which 200 specimen were identified to belong to 46 agaric species (Asef \& Etemad 2016).

Collection and identification of native wild mushrooms may have various applications in taxonomical and ethnomycological studies, documentation related to the traditional use of mushrooms, and local markets (Kamalebo et al. 2018). It may even promote further investigations for the domestication of wild edible mushrooms not yet cultivated in a country (Semwal et al. 2014). However, it does not necessarily address the challenge with production of fruiting bodies and pure culture of wild mushrooms. Adaptation of wild mushrooms in locally available lignocellulosic substrates to produce fruiting bodies is essential to ensure ongoing reliability and reproducibility of research on biomedical and biochemical aspects of wild mushrooms (Lindequist et al. 2005). To the best of our knowledge, limited information is available regarding characterization of growth of Iranian wild-growing macrofungi in solid media, spawn, or locally available substrates, although unpublished efforts might have been made to this end. Following the previous experiences with domestication of Iranian wild-growing Enoki (Flammulina velutipes) (Rezaeian \& Pourianfar 2017), this study aimed to evaluate adaptation of 40 mushroom isolates indigenous to Mazandaran province (Northern Iran) in solid media, spawn, and locally available lignocellulosic substrate.

\section{Materials \& Methods}

\section{Mushroom samples}

Totally 40 isolates of wild-growing mushrooms were provided as mycelia cultures (in media) as indicated in Table 1. These isolates were selected from a collection of wild mushrooms that have been collected from Mazandaran province (located in Northern Iran that lies along the Caspian Sea) during the year 2015-2017 and have been deposited at Microorganisms Bank of Iranian Biological Resource Center (IBRC). Identification of the collection of wild mushrooms has been performed by experts of IBRC based on morphological and microscopic characteristics. In addition, the strain names assigned to each species utilized in this study were provided by IBRC (indicated in parentheses after the scientific name of each isolate). 


\section{Mycelial culture}

Specific media for growing mycelia of the samples included Potato Dextrose Agar (PDA) and compost extract agar (CEA). PDA was purchased from Merck (Darmstadt, Germany). CEA was manually prepared as detailed by Masoumi et al. (2015). Three hundred grams of the pasteurized fresh compost of the white button mushroom was boiled in one liter of distilled water for one hour, followed by centrifugation (Hermle Labor Technik GmbH, Germany) at $5000 \mathrm{rpm}$ for five minutes. The upper phase was collected, filtered and used as the compost extract to be mixed with $0.5 \%$ agar (Merck, Darmstadt, Germany) v/w to serve as a solid media. In all the experimental steps, deionized water was utilized to prepare solutions and buffers. Both PDA and CEA media were sterilized for 20 minutes at $121^{\circ} \mathrm{C}$ and then aseptically poured into gamma-sterilized 8-cm plastic petri dishes. The solid media was inoculated with $1 \mathrm{~cm}^{2}$ disks of the leading edges of mushroom mycelia. The cultures were then incubated in the dark for two weeks at $25 \pm 2^{\circ} \mathrm{C}$, after which the daily mycelial growth rate was evaluated (Rezaeian \& Pourianfar 2017). Two vertical lines were drawn on each petri dish and four points of mycelia extensions at perpendicular angles were marked. With the aid of a caliper, means of readings of the four points were measured daily. After 14 days, the radial growth rate was calculated based on daily averages and expressed as millimeter per day. Three independent replicates were made for each isolate in each media.

\section{Spawn preparation}

Two types of spawn were evaluated, including grain-based and wood-based substrates. Grainbased substrate was prepared using a mixture of boiled wheat grains supplemented with $1 \% \mathrm{w} / \mathrm{v}$ $\mathrm{CaCo}_{3}$ and $2 \%$ w/v CaSO 4 , as described by Ghanbari et al. (2015). Wood based-spawn was made of wheat grains enriched with $25 \%$ wood chips, being conditioned with $1 \% \mathrm{w} / \mathrm{v} \mathrm{CaCO}_{3}$ and $2 \%$ $\mathrm{w} / \mathrm{v} \mathrm{CaSO} 4$ (Erkel 2009). Polypropylene bags of $18 \times 32.5 \mathrm{~cm}$ were then filled with one kilograms of each substrate and packed tightly followed by sterilization at 15 psi, $121^{\circ} \mathrm{C}$ for 120 minutes. Inoculation was performed using pure actively growing mycelia. The spawn bags were incubated in $25^{\circ} \pm 2^{\circ} \mathrm{C}$ until they were fully colonized by mushroom mycelia (over a period of two months). Three independent replicates were made for each isolate in each type of spawn.

\section{Fruiting test}

Wood chips (obtained from Populus alba) were utilized as the main substrate in order to test fructification of the wild strains, based on the assumption that the collected mushroom isolates were wood-decay fungi growing in Mazandaran forests. Wood chips were soaked for 18 hours followed by mixing with $15 \%$ wheat bran and $3 \% \mathrm{CaCO}_{3}$ in $600 \mathrm{~g}$ cellophane plastic bags. Then, the bags were sterilized at $15 \mathrm{psi}$ and $121^{\circ} \mathrm{C}$ for $120 \mathrm{~min}$. Spawn rate and environmental conditions were adapted from the previous experiments with wild-growing medicinal mushroom Flammulina velutipes (Rezaeian \& Pourianfar 2017). Thirty grams of the fresh spawn (containing actively growing mycelia) aseptically inoculated one $\mathrm{kg}$ of fresh raw substrate, followed by sealing and transferring to the cultivation room. For some isolates, due to the lack of growth in spawn, a couple of 3-cm plug of newly grown mycelium in petri dishes were directly used to inoculate the substrate. All the bags were placed in a dark room at $25^{\circ} \mathrm{C} \pm 2^{\circ} \mathrm{C}$ until completion of the mushroom vegetative phase. They were then categorized into two different temperature treatments: (i) pre-cooling in a refrigerator at $7-9^{\circ} \mathrm{C}$ for $8-10$ days before transferring to the cropping room; (ii) transferring to the cropping room directly without the pre-cooling treatment. Three independent replicates with two sub-samples were made for each isolate in each temperature treatment. The relative humidity of 85 to $90 \%$ and a room temperature of $18-20^{\circ} \mathrm{C}$ were maintained in the cropping room for fructification.

\section{Statistical analysis}

All the experiment were independently repeated three times. JMP version 8 was utilized to conduct statistical analyses and ANOVA tests. Means were compared using Duncan's multiple range test with a significance level ( $p$ value) of 0.05 . 


\section{Results}

\section{Adaptation of mycelia of wild mushrooms in solid media}

Over a period of 14 days, mycelia of all the wild mushroom isolates grew to some extent in PDA (Figs 1,2) and CEA media (Figs 3, 4) and were expressed as average daily mycelial growth (mm per day). Overall, there were significant differences in growth rates of mycelia between the media and between the isolates as well $(p<0.01)$. Table 1 illustrates the average daily mycelial growth rates (mm per day) obtained for each isolate in each media.

Among the tested isolates, Irpex lacteus (GPS 005) displayed the highest daily mycelial growth rate in both media, including $8.37 \pm 0.05$ and $8.2 \pm 0.07 \mathrm{~mm}$ per day in PDA (Fig. 1) and CEA (Fig. 3), respectively (Table 1). The findings also revealed that average daily growth rates of mycelia recorded in PDA for Donkia pulcherrima (Neka 24D) (Fig. 1), Daedaleopsis sp. (Royan 8) (Fig. 2), Lenzites tricolor (GPS 180) (Fig. 2), Trametes sp. (GPS 122), Trametes versicolor (GPS 107) and Stereum hirsutum (Darabkola 1) (Fig. 1) were significantly higher as compared with those in CEA medium $(p<0.01$ ) (Figs 3, 4). Donkia pulcherrima (Neka 24D) showed a daily growth rate of $4.34 \pm 0.22$ (mm per day) in PDA, while the records in the same media for Daedaleopsis sp. (Royan 8), Lenzites tricolor (GPS 180), and Stereum hirsutum (Darabkola 1) were 3.23 \pm 0.07 , $3.81 \pm 0.12$, and $4.82 \pm 0.04$ (mm per day), respectively (Table 1$)(p<0.05)$.

Hypholoma fasciculare (GPS 176) (Fig. 4), Cyclocybe sp. (Darabkola-20) (Fig. 4), Hypholoma sp. (GPS 106) (Fig. 4), Coprinellus disseminates (Nur 9) (Fig. 3), and Psathyrella candolleana (Nur 10) (Fig. 4) had higher daily radial growth rates in CEA as compared with PDA medium. Coprinellus disseminates (Nur 9) displayed an average daily mycelial growth rate of $5.66 \pm 0.21$ ( $m m$ per day) in CEA. The average daily mycelial growth rates in the same media for Cyclocybe sp. (Darabkola-20), Hypholoma sp. (GPS 177), and Psathyrella candolleana (Nur 10) were 3.29 $\pm 0.07,1.69 \pm 0.58$, and 3.45 \pm 0.02 (mm per day), respectively $(p<0.01)$ (Table 1 ).

A high daily growth rate (more than $4 \mathrm{~mm}$ per day) was also observed in mycelia of Trametes sp. (GPS 130), Trametes sp. (Neka 25-2), Trametes sp. (GPS 179), Marasmiellus sp. (GPS 30), Hydnopolyporus fimbriatus (GPS 29), Trametes sp. (Darabkola 13), Trametes versicolor (GPS 007), Trametes gibbosa (Nur 8), Trametes gibbosa (GPS22), Irpex lacteus (GPS 005), Trametes hirsuta (GPS 042), Ganoderma sp. (GPS 52), Trametes gibbosa (GPS 63) and Trametes sp.(GPS 119) .However, there was no significant difference between PDA (Figs 1, 2) and CEA (Figs 3, 4) in terms of mycelial growth rates of these isolates $(p \geq 0.05)$ (Table 1$)$. In addition, no significant difference $(p \geq 0.05)$ was observed between the media in the average daily mycelial growth of Macrolepiota konradii (GPS 101), Hohenbuehelia auriscalpium (GPS 196), Armillaria sp. (GPS 173), Ganoderma sp. (GPS 17), and Ganoderma sp. (Bozchaft 2), all of which showed a slow daily growth rate less than $2 \mathrm{~mm}$ per day in both media, PDA (Figs 1, 2) and CEA (Figs 3, 4) (Table 1).

Table 1 Growth characterization and fructification of wild mushrooms

\begin{tabular}{|c|l|l|l|l|l|l|}
\hline & \multirow{2}{*}{ Species (strain) } & $\begin{array}{l}\text { Average daily mycelial } \\
\text { growth (mm per day) }\end{array}$ & $\begin{array}{l}\text { Period of spawn } \\
\text { development (day) }\end{array}$ & \multirow{2}{*}{ Fructification } \\
\cline { 3 - 6 } & & CEA & PDA & $\begin{array}{l}\text { Wheat } \\
\text { grains }\end{array}$ & $\begin{array}{l}\text { Wood } \\
\text { chips }\end{array}$ & \\
\hline 1 & Trametes sp. (Neka 25-2) & $3.51 \pm 0.04^{\mathrm{a}}$ & $3.63 \pm 0.28^{\mathrm{a}}$ & $14 \pm 1^{\mathrm{a}}$ & $25 \pm 1^{\mathrm{b}}$ & No \\
\hline 2 & Trametes sp. (GPS 179) & $4.1 \pm 0.11^{\mathrm{a}}$ & $4.13 \pm 0.08^{\mathrm{a}}$ & $15 \pm 1^{\mathrm{a}}$ & $25 \pm 1^{\mathrm{b}}$ & No \\
\hline 3 & $\begin{array}{l}\text { Hydnopolyporus fimbriatus } \\
\text { (GPS 29) }^{*}\end{array}$ & $5.43 \pm 0.05^{\mathrm{a}}$ & $5.51 \pm 0.01^{\mathrm{a}}$ & No growth & $28 \pm 2^{\mathrm{b}}$ & No \\
\hline 4 & Trametes hirsuta (GPS 042 ) & $4.8 \pm 0.08^{\mathrm{a}}$ & $4.64 \pm 0.21^{\mathrm{a}}$ & No growth $^{\mathrm{a}}$ & $25 \pm 1^{\mathrm{b}}$ & No \\
\hline 5 & Trametes gibbosa (GPS 63) & $4.76 \pm 0.12^{\mathrm{a}}$ & $4.77 \pm 0.15^{\mathrm{a}}$ & $14 \pm 1^{\mathrm{a}}$ & $26 \pm 1^{\mathrm{b}}$ & No \\
\hline 6 & Trametes sp. (GPS 119) & $4.91 \pm 0.14^{\mathrm{a}}$ & $5.02 \pm 0.13^{\mathrm{a}}$ & $14 \pm 1^{\mathrm{a}}$ & $25 \pm 1^{\mathrm{b}}$ & No \\
\hline 7 & Irpex sp. (GPS 146) & $4.9 \pm 0.12^{\mathrm{a}}$ & $6.67 \pm 0.06^{\mathrm{b}}$ & $18 \pm 3^{\mathrm{a}}$ & $28 \pm 2^{\mathrm{b}}$ & No \\
\hline 8 & Trametes sp. (GPS 122) & $4.6 \pm 0.22^{\mathrm{a}}$ & $5.13 \pm 0.08^{\mathrm{a}}$ & $15 \pm 1^{\mathrm{a}}$ & $25 \pm 3^{\mathrm{b}}$ & No \\
\hline 9 & Irpex lacteus (GPS 005) & $8.2 \pm 0.07^{\mathrm{a}}$ & $8.37 \pm 0.05^{\mathrm{b}}$ & $17 \pm 2^{\mathrm{a}}$ & $27 \pm 2^{\mathrm{b}}$ & No \\
\hline 10 & Trametes sp. (GPS 130) & $3.72 \pm 0.17^{\mathrm{a}}$ & $4.16 \pm 0.06^{\mathrm{a}}$ & $14 \pm 1^{\mathrm{a}}$ & $27 \pm 2$ & Yes \\
\hline
\end{tabular}


Table 1 Continued.

\begin{tabular}{|c|c|c|c|c|c|c|}
\hline & \multirow{2}{*}{ Species (strain) } & \multicolumn{2}{|c|}{$\begin{array}{l}\text { Average daily mycelial } \\
\text { growth (mm per day) }\end{array}$} & \multicolumn{2}{|c|}{$\begin{array}{l}\text { Period of spawn } \\
\text { development (day) }\end{array}$} & \multirow{2}{*}{ Fructification } \\
\hline & & CEA & PDA & $\begin{array}{l}\text { Wheat } \\
\text { grains }\end{array}$ & $\begin{array}{l}\text { Wood } \\
\text { chips }\end{array}$ & \\
\hline 11 & Trametes gibbosa (GPS 22) & $3.22 \pm 0.039^{\mathrm{a}}$ & $4.73 \pm 1.19^{\mathrm{a}}$ & $14 \pm 1^{\mathrm{a}}$ & $25 \pm 1^{b}$ & No \\
\hline 12 & Ganoderma sp. (GPS 37) & $3.13 \pm 0.04^{\mathrm{a}}$ & $4.77 \pm 0.11^{\mathrm{b}}$ & $14 \pm 1^{\mathrm{a}}$ & $25 \pm 1^{b}$ & No \\
\hline 13 & Ganoderma sp. (GPS 38) & $3.59 \pm 0.11^{\mathrm{a}}$ & $4.25 \pm 0.03^{\mathrm{b}}$ & No growth ${ }^{\mathrm{a}}$ & $26 \pm 2^{b}$ & No \\
\hline 14 & Ganoderma sp. (GPS 52) & $3.91 \pm 0.24^{\mathrm{a}}$ & $4.3 \pm 0.48^{\mathrm{a}}$ & No growth ${ }^{\mathrm{a}}$ & $26 \pm 1^{b}$ & No \\
\hline 15 & $\begin{array}{l}\text { Trametes versicolor } \\
\text { (GPS 107) }\end{array}$ & $3.86 \pm 0.12^{\mathrm{a}}$ & $5.07 \pm 0.14^{\mathrm{b}}$ & $14 \pm 1^{\mathrm{a}}$ & $26 \pm 1^{b}$ & No \\
\hline 16 & $\begin{array}{l}\text { Stereum hirsutum } \\
\text { (Darabkola 1) }\end{array}$ & $2.77 \pm 0.12^{\mathrm{a}}$ & $4.82 \pm 0.04^{\mathrm{b}}$ & No growth ${ }^{\mathrm{a}}$ & $27 \pm 1^{b}$ & No \\
\hline 17 & $\begin{array}{l}\text { Donkia pulcherrima } \\
\text { (Neka 24D) }\end{array}$ & $0.66 \pm 0.68^{\mathrm{a}}$ & $4.34 \pm 0.22^{\mathrm{b}}$ & No growth ${ }^{\mathrm{a}}$ & No growth ${ }^{\mathrm{a}}$ & Yes \\
\hline 18 & $\begin{array}{l}\text { Coprinellus disseminates } \\
\text { (Nur 9) }\end{array}$ & $5.66 \pm 0.209^{\mathrm{a}}$ & $3.42 \pm 0.08^{b}$ & $22 \pm 3^{a}$ & $31 \pm 2^{b}$ & No \\
\hline 19 & Marasmiellus sp. (GPS 30) & $3.04 \pm 0.021^{\mathrm{a}}$ & $3.23 \pm 0.18^{\mathrm{a}}$ & $19 \pm 2^{\mathrm{a}}$ & $29 \pm 2^{b}$ & No \\
\hline 20 & Trametes sp. (Darabkola 13) & $3.95 \pm 0.14^{\mathrm{a}}$ & $4.08 \pm 0.11^{\mathrm{a}}$ & $14 \pm 0^{\mathrm{a}}$ & $22 \pm 1$ & Yes \\
\hline 21 & Trametes gibbosa (Nur 8) & $3.48 \pm 0.023^{\mathrm{a}}$ & $3.48 \pm 0.13^{\mathrm{a}}$ & $14 \pm 2^{\mathrm{a}}$ & $25 \pm 2^{b}$ & No \\
\hline 22 & $\begin{array}{l}\text { Trametes versicolor } \\
\text { (GPS 007) }\end{array}$ & $3.29 \pm 0.04^{\mathrm{a}}$ & $3.23 \pm 0.05^{\mathrm{a}}$ & $14 \pm 2^{\mathrm{a}}$ & $28 \pm 2^{\mathrm{b}}$ & No \\
\hline 23 & Lenzites tricolor (GPS 180) & $2.91 \pm 0.09^{\mathrm{a}}$ & $3.81 \pm 0.12^{\mathrm{b}}$ & $14 \pm 2^{\mathrm{a}}$ & $27 \pm 2^{b}$ & Yes \\
\hline 24 & Daedaleopsis sp. (Royan 8) & $2.02 \pm 0.13^{\mathrm{a}}$ & $3.23 \pm 0.07^{\mathrm{b}}$ & $15 \pm 3^{\mathrm{a}}$ & $31 \pm 3^{b}$ & Yes \\
\hline 25 & $\begin{array}{l}\text { Psathyrella candolleana } \\
\text { (Nur 10) }\end{array}$ & $3.45 \pm 0.02^{\mathrm{a}}$ & $2.23 \pm 0.01^{\mathrm{b}}$ & $18 \pm 1^{\mathrm{a}}$ & $29 \pm 2^{b}$ & No \\
\hline 26 & $\begin{array}{l}\text { Cyclocybe sp. } \\
\text { (Darabkola 20) }\end{array}$ & $3.29 \pm 0.07^{\mathrm{a}}$ & $2.94 \pm 0.09^{\mathrm{b}}$ & $14 \pm 2^{\mathrm{a}}$ & $25 \pm 2^{b}$ & Yes \\
\hline 27 & $\begin{array}{l}\text { Ganoderma tsugae } \\
\text { (GPS 186) }\end{array}$ & $2.19 \pm 0.54^{\mathrm{a}}$ & $2.22 \pm 0.29^{\mathrm{b}}$ & $14 \pm 0^{\mathrm{a}}$ & $25 \pm 2^{b}$ & Yes \\
\hline 28 & $\begin{array}{l}\text { Fomes fomentarius } \\
\text { (GPS 131) }\end{array}$ & $2.79 \pm 0.42^{\mathrm{a}}$ & $3.1 \pm 0.38^{b}$ & $14 \pm 1^{\mathrm{a}}$ & $25 \pm 1^{b}$ & No \\
\hline 29 & $\begin{array}{l}\text { Pholiota aurivella } \\
\text { (GPS 142) }\end{array}$ & $2.52 \pm 0.39^{\mathrm{a}}$ & $2.01 \pm 0.06^{\mathrm{a}}$ & $19 \pm 1^{\mathrm{a}}$ & $26 \pm 1^{b}$ & Yes \\
\hline 30 & $\begin{array}{l}\text { Ganoderma sp. } \\
\text { (Neka 25-1) }\end{array}$ & $2.84 \pm 0.03^{\mathrm{a}}$ & $2.93 \pm 0.35^{\mathrm{a}}$ & $14 \pm 2^{\mathrm{a}}$ & $29 \pm 1^{b}$ & No \\
\hline 31 & $\begin{array}{l}\text { Lycoperdon pyriforme } \\
\text { (GPS 158)* }\end{array}$ & $1.23 \pm 0.11^{\mathrm{a}}$ & $1.17 \pm 0.07^{\mathrm{a}}$ & $27 \pm 2^{\mathrm{a}}$ & $24 \pm 2^{\mathrm{a}}$ & No \\
\hline 32 & Leucoagaricus sp. (Royan 1) & $2.08 \pm 0.15^{\mathrm{a}}$ & $1.17 \pm 0.08^{\mathrm{b}}$ & $25 \pm 2^{\mathrm{a}}$ & $32 \pm 3^{b}$ & No \\
\hline 33 & Ganoderma sp. (Bozchaft 2) & $1.62 \pm 0.52^{\mathrm{a}}$ & $1.69 \pm 0.19^{\mathrm{a}}$ & $14 \pm 1^{\mathrm{a}}$ & $30 \pm 2^{b}$ & No \\
\hline 34 & Ganoderma sp. (GPS 17) & $1.63 \pm 0.61^{\mathrm{a}}$ & $1.46 \pm 0.37^{\mathrm{a}}$ & No growth $^{\mathrm{a}}$ & $25 \pm 2^{b}$ & No \\
\hline 35 & $\begin{array}{l}\text { Hypholoma fasciculare } \\
\text { (GPS 176) }\end{array}$ & $1.23 \pm 0.11^{\mathrm{a}}$ & $0.8 \pm 0.007^{b}$ & $25 \pm 2^{\mathrm{a}}$ & $25 \pm 1^{\mathrm{b}}$ & No \\
\hline 36 & Hypholoma sp. (GPS 177) $^{*}$ & $1.69 \pm 0.58^{\mathrm{a}}$ & $0.64 \pm 0.08^{\mathrm{b}}$ & $17 \pm 2^{\mathrm{a}}$ & $25 \pm 2^{b}$ & No \\
\hline 37 & Hypholoma sp.(GPS 106) & $1.81 \pm 0.04^{\mathrm{a}}$ & $0.64 \pm 0.15^{\mathrm{b}}$ & $14 \pm 1^{\mathrm{a}}$ & $35 \pm 3^{b}$ & No \\
\hline 38 & $\begin{array}{l}\text { Hohenbuehelia auriscalpium } \\
\text { (GPS 196) }\end{array}$ & $0.55 \pm 0.54^{\mathrm{a}}$ & $0.26 \pm 0.09^{\mathrm{b}}$ & No growth $^{\mathrm{a}}$ & No growth ${ }^{\mathrm{a}}$ & No \\
\hline 39 & Armillaria sp. (GPS 173) & $0.52 \pm 0.10^{\mathrm{a}}$ & $0.44 \pm 0.06^{\mathrm{a}}$ & $38 \pm 2^{\mathrm{a}}$ & $45 \pm 4^{\mathrm{b}}$ & No \\
\hline 40 & $\begin{array}{l}\text { Macrolepiota konradii } \\
\text { (GPS 101) }\end{array}$ & $1.59 \pm 0.02^{\mathrm{a}}$ & $1.12 \pm 0.04^{\mathrm{a}}$ & $23 \pm 3^{a}$ & $27 \pm 3^{\mathrm{a}}$ & No \\
\hline
\end{tabular}

The wild mushroom species in Table 1 have been identified by Microorganisms Bank of Iranian Biological Resource Center (IBRC), Tehran, Iran. The strain names assigned to these species (given in parentheses) have been provided by the same center. Mycelial growth was measured in Potato Dextrose Agar (PDA) or Compost Extract Agar (CEA). The data of mycelial growth were collected over 14 days and expressed as average daily mycelial growth rate (millimeter per day). The days indicated for spawn development are the period of time required by the wild mushrooms mycelia to fully colonize wheat grains or wood chips. Statistical comparisons have been made between different solid media and between different spawn substrates within each row (for each 
mushroom strain). Means followed by the same superscript lower-case letters are not significantly different (Duncan's multiple range test, $p<0.05$ ). Asterisks indicate some of the most significant differences among the mushroom strains, determined based on main effects of each strain on mycelial growth averaging across both PDA and CEA media (Duncan's multiple range test, $p<0.05$ ).
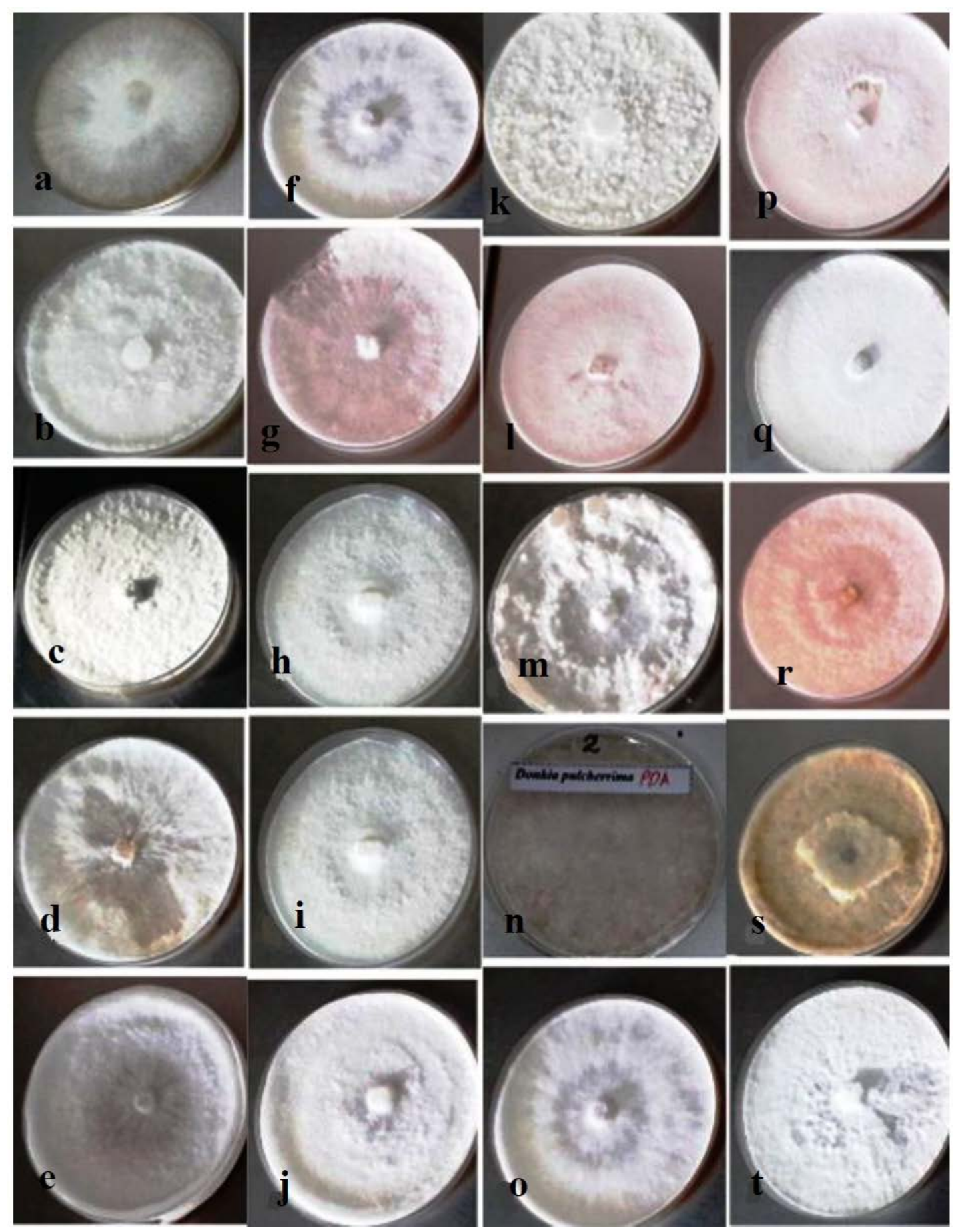

Fig. 1 - Mycelial growth of wild mushroom isolates in PDA. Panels a-t illustrate PDA cultures of mycelia of Trametes sp. (Neka 25-2), Hydnopolyporus fimbriatus (GPS 29), Trametes gibbosa (GPS 63), Irpex sp. (GPS 146), Irpex lacteus (GPS 005), Trametes sp. (GPS 179), Trametes hirsuta (GPS 042 ), Trametes sp. (GPS 119), Trametes sp. (GPS 122), Trametes sp. (GPS 130), Trametes gibbosa (GPS 22), Ganoderma sp. (GPS 38), Trametes versicolor (GPS 107), Donkia pulcherrima (Neka 24D), Marasmiellus sp. (GPS 30), Ganoderma sp. (GPS 37), Ganoderma sp. (GPS 52), Stereum hirsutum (Darabkola 1), Coprinellus disseminates (Nur 9) and Trametes sp. (Darabkola 13) respectively. Photographs were taken with a digital camera on the fourteenth day of mycelial growth in the first subculture. Each isolate was subjected to three independent replications. 

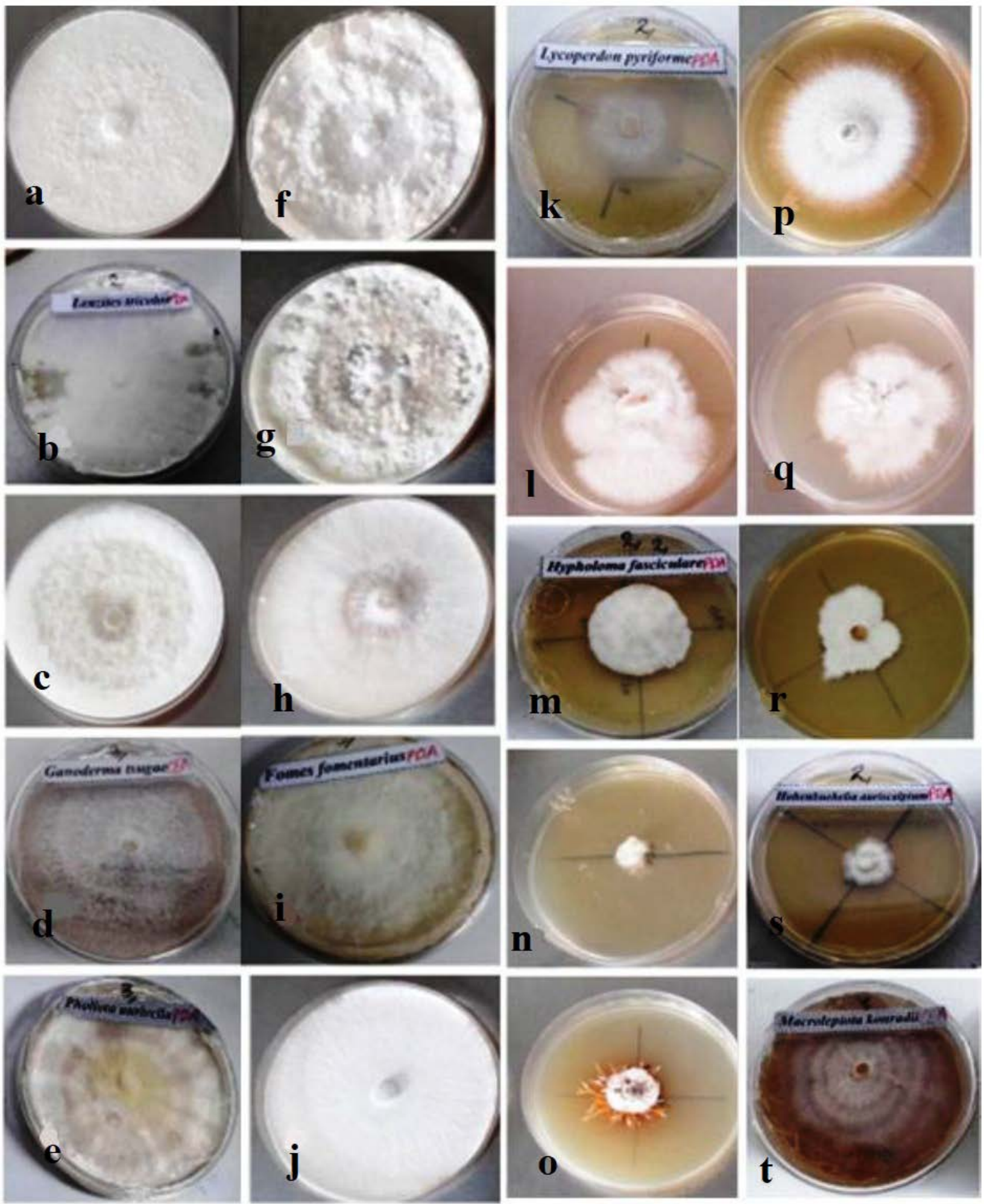

Fig. 2 - Mycelial growth of wild mushroom isolates in PDA. Panels a-t illustrate PDA cultures of mycelia of Trametes gibbosa (Nur 8), Lenzites tricolor (GPS 180), Psathyrella candolleana (Nur 10), Ganoderma tsugae (GPS 186), Pholiota aurivella (GPS 142), Trametes versicolor (GPS 007), Daedaleopsis sp. (Royan 8), Cyclocybe sp. (Darabkola 20), Fomes fomentarius (GPS 131), Ganoderma sp. (Neka 25-1), Lycoperdon pyriforme (GPS 158), Ganoderma sp. (Bozchaft 2), Hypholoma fasciculare (GPS 176), Hypholoma sp. (GPS 106), Armillaria sp. (GPS 173) , Leucoagaricus sp. (Royan 1), Ganoderma sp. (GPS 17), Hypholoma sp. (GPS 177), Hohenbuehelia auriscalpium (GPS 196) and Macrolepiota konradii (GPS 101) respectively. Photographs were taken with a digital camera on the fourteenth day of mycelial growth in the first subculture. Each isolate was subjected to three independent replications. 

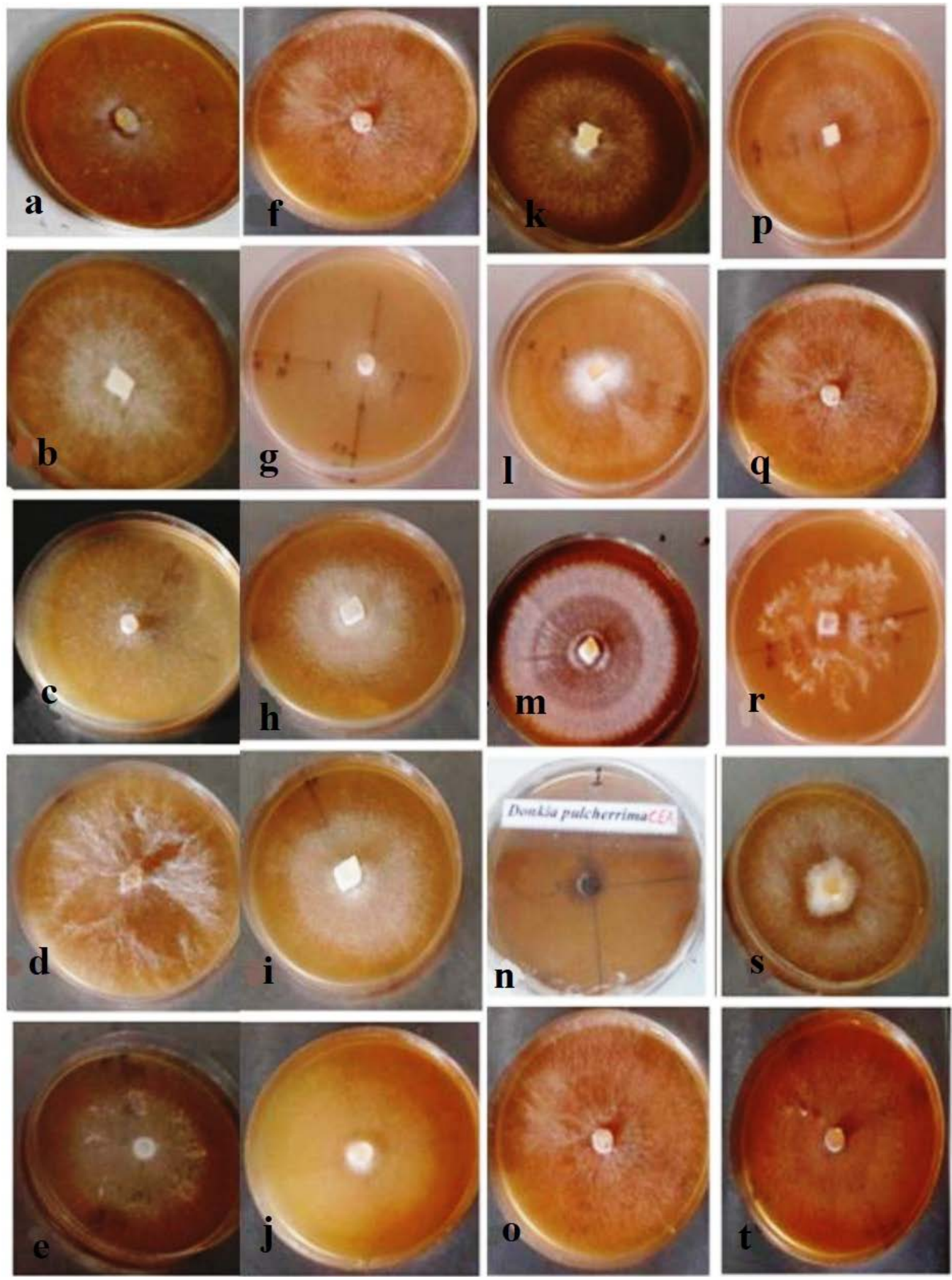

Fig. 3 - Mycelial growth of wild mushroom isolates in CEA. Panels a-t illustrate CEA cultures of mycelia of Trametes sp. (Neka 25-2), Hydnopolyporus fimbriatus (GPS 29), Trametes gibbosa (GPS 63), Irpex sp. (GPS 146), Irpex lacteus (GPS 005), Trametes sp. (GPS 179), Trametes hirsuta (GPS 042 ), Trametes sp. (GPS 119), Trametes sp. (GPS 122), Trametes sp. (GPS 130), Trametes gibbosa (GPS 22), Ganoderma sp. (GPS 38), Trametes versicolor (GPS 107), Donkia pulcherrima (Neka 24D), Marasmiellus sp. (GPS 30), Ganoderma sp. (GPS 37), Ganoderma sp. (GPS 52), Stereum hirsutum (Darabkola 1), Coprinellus disseminates (Nur 9) and Trametes sp. (Darabkola 13) respectively. Photographs were taken with a digital camera on the fourteenth day of mycelial growth in the first subculture. Each isolate was subjected to three independent replications. 

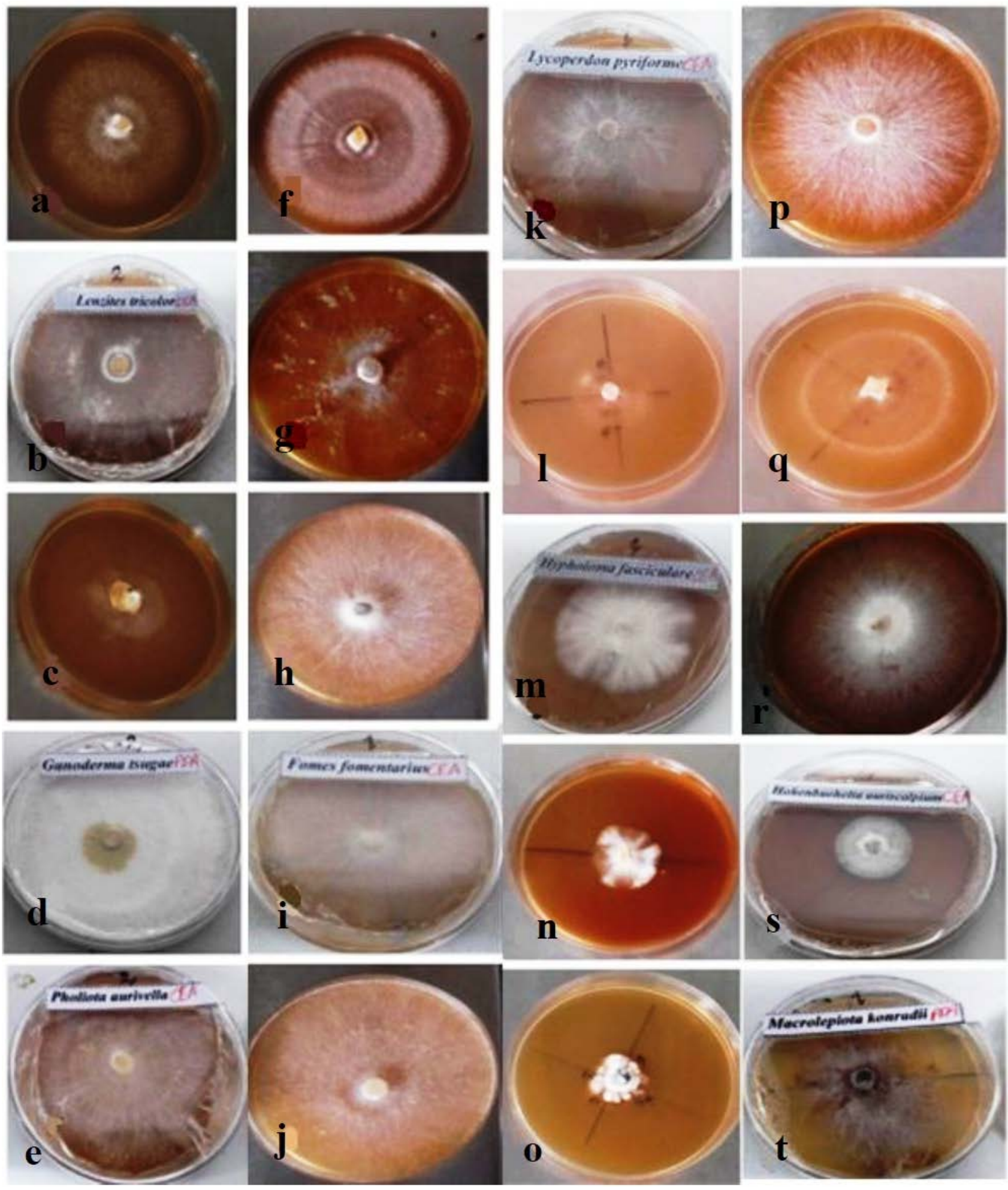

Fig. 4 - Mycelial growth of wild mushroom isolates in CEA. Panels a-t illustrate CEA cultures of mycelia of Trametes gibbosa (Nur8), Lenzites tricolor (GPS 180), Psathyrella candolleana (Nur 10), Ganoderma tsugae (GPS 186), Pholiota aurivella (GPS 142), Trametes versicolor (GPS 007), Daedaleopsis sp. (Royan 8), Cyclocybe sp. (Darabkola 20), Fomes fomentarius (GPS 131), Ganoderma sp. (Neka 25-1), Lycoperdon pyriforme (GPS 158), Ganoderma sp. (Bozchaft 2), Hypholoma fasciculare (GPS 176), Hypholoma sp. (GPS 106), Armillaria sp. (GPS 173) , Leucoagaricus sp. (Royan 1), Ganoderma sp. (GPS 17), Hypholoma sp. (GPS 177), Hohenbuehelia auriscalpium (GPS 196) and Macrolepiota konradii (GPS 101) respectively. Photographs were taken with a digital camera on the fourteenth day of mycelial growth in the first subculture. Each isolate was subjected to three independent replications. 


\section{Colonization of spawn by wild mushrooms}

Overall, most of the wild mushrooms filled wood spawn for 24-45 days after inoculation, which was significantly longer than the period observed in grain spawn $(p<0.01)$ (Table 1$)$. Mycelia of Lenzites tricolor (GPS 180) (Fig. 5a), Trametes sp. (GPS 119), Daedaleopsis sp. (Royan 8) (Fig. 5b), Trametes versicolor (GPS 107), Trametes sp. (GPS 122), Trametes sp. (GPS 130) (Fig. 5e), Ganoderma tsugae (GPS 186) (Fig. 5g), Trametes sp. (Darabkola 13), Trametes gibbosa (Nur 8), Ganoderma sp. (Neka 25-1), Ganoderma sp. (GPS 37), Ganoderma sp. (Bozchaft 2), Hypholoma sp. (GPS 106), Trametes gibbosa (GPS 63), Trametes gibbosa (GPS 22), Cyclocybe sp. (Darabkola 20) (Fig. 5f), Trametes sp. (GPS 179), Trametes versicolor (GPS 007), Trametes sp. (Neka 25-2), and Fomes fomentarius (GPS 131) completely colonized wheat grains for 14-15 days after inoculation. The spawn-growing periods for Pholiota aurivella (GPS 142) (Fig. 5c), Macrolepoita konradii (GPS 101), Hypholma fasciculare (GPS 176) and Lycopedron pyriforme (GPS 158) were 19, 23, 25, and 27 days, respectively $(p<0.05)$. No growth of mycelia in wheat grains or wood chips ( $p>0.05$ ) was observed for Donkia pulcherrima (Neka 24D) (Fig. 5d) and Hohenbuehelia auriscapium (GPS 196) (Fig. 5h) over one month of incubation (Table 1). Thus, mycelia plugs of these mushrooms grown in the solid media were utilized to inoculate substrate directly.

Observations also showed that mycelium of Hydnopolyporus fimbriatus (GPS 29), Ganoderma sp. (GPS 17), Ganoderma sp. (GPS 38), Ganoderma sp. (GPS 52), Stereum hirsutum (Darabkola 1), and Trametes hirsuta (GPS 042) did not grow in grain spawn, while they colonized wood chips $25-28$ days after inoculation $(p<0.01)$ (Table 1$)$.

\section{Adaptation of wild strains in lignocellulosic agro-wastes}

The vegetative phase of all the tested wild mushrooms in the substrate (mycelial running) was completed 17-22 days after inoculation, indicating insignificant variations among the mushroom isolates $(p \geq 0.05)$. However, fructification was observed only among fully colonized substrates pre-conditioned in the refrigerator, while the substrates that did not pass this period produced no fruiting bodies. Of the substrates pre-conditioned in the refrigerator, eight isolates belonging to seven different genera successfully fructified, including Pholiota aurivella (GPS 142), Lenzites tricolor (GPS 180), Ganoderma tsugae (GPS 186), Cyclocybe sp. (Darabkola 20), Trametes sp. (GPS 130), Trametes sp. (Darabkola 13), Daedaleopsis sp. (Royan 8), and Donkia pulcherrima (Neka 24D) (Figs 6, 7).

It took about three months (after transferring the fully colonized substrates to the cropping room) for Ganoderma tsugae (GPS 186) to develop first pinheads, lasted for more than 35 days. During the fruiting bodies development, the cap color changed from white to yellow and brown (Fig. 6a, b). Besides, the first pinheads of Daedaleopsis sp. (Royan 8) emerged 42 days after transferring the fully colonized substrates to the cropping room, lasted for up to 35 days during which the mature cap showed a brown cap (Fig. 6c, d). Observations also showed that the first primordia of Donkia pulcherrima (Neka 24D) appeared 90 days after transferring bags to the cropping room, followed by maturation of hairy caps during one week that lasted for three days (Fig. 6e, f).

Daily observations also showed that Trametes sp. (strains of GPS 130 and Darabkola 13) and Cyclocybe sp. (Darabkola 20) developed pinheads 28 days after transferring to the cropping room. It took 25-30 days (after appearance of pinheads) for fruiting bodies of Trametes sp. isolates (strains of GPS 130 and Darabkola 13) to mature. Reproductive structure of both isolates of Trametes sp. lasted 25 days during which fruiting bodies showed color changes from a bright cream to orange brown (like turkey tail) (Fig. 7a, b). Cyclocybe sp. (Darabkola 20) fruiting bodies showed caps similar to those of the button mushroom (Agaricus bisporus), appeared one week after the development of pinheads and lasted about 3 days (Fig. 7c, d).

Pholiota aurivella (GPS 142) and Lenzites tricolor (GPS 180) produced early reproductive structures 50 days after transferring the fully colonized substrates to the cropping room. The reproductive structures of Pholiota aurivella (GPS 142) emerged in the form of orange-colored 
caps as clusters on the substrate (Fig. 7 e, f), while those of Lenzites tricolor (GPS 180), appeared as separate multiple fruiting bodies on the substrate (Fig. $7 \mathrm{~g}, \mathrm{~h}$ ).

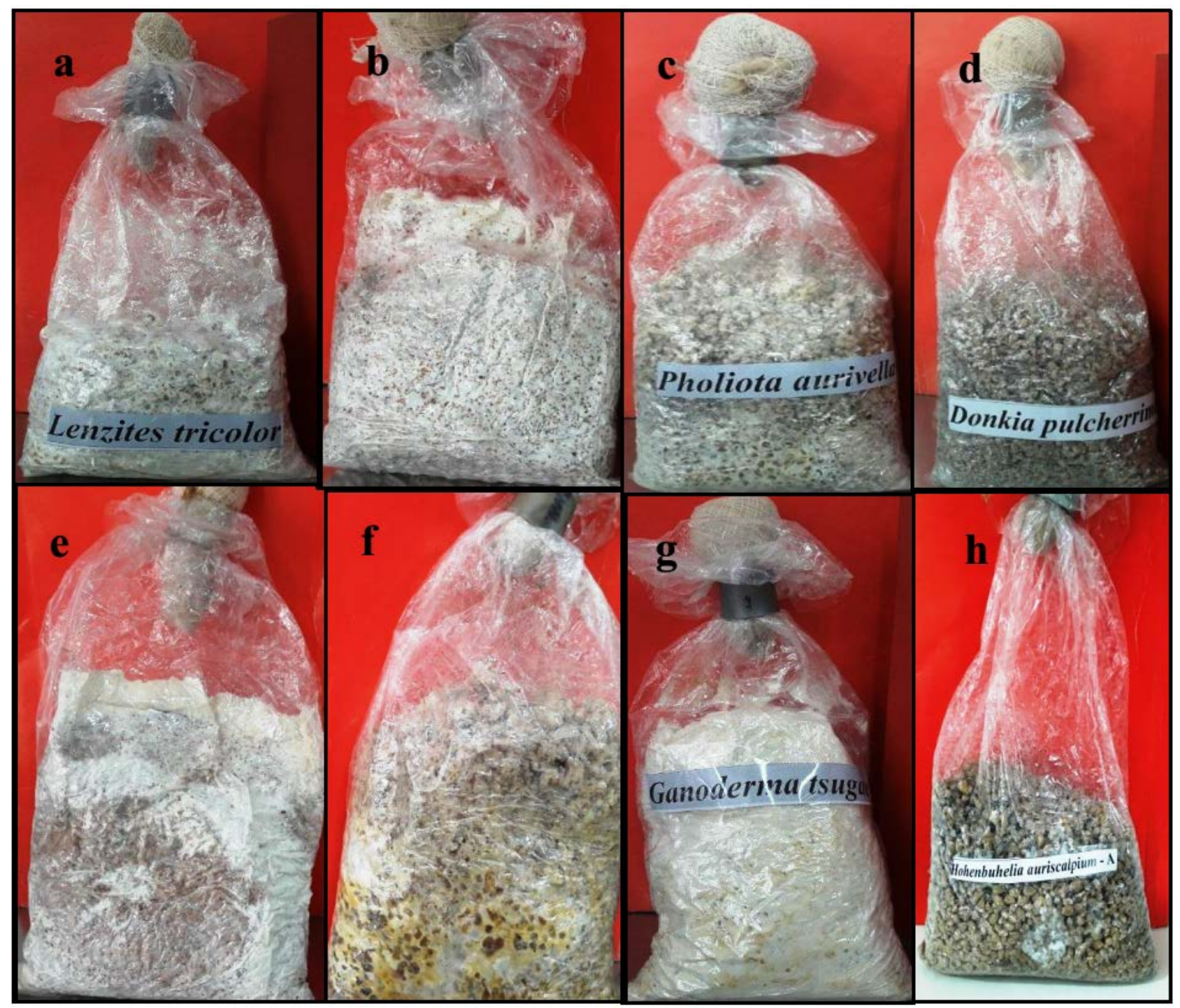

Fig. 5 - Differences in the growth of spawn between selected wild mushrooms in wheat grains. Panels a-h illustrate grain spawn of Lenzites tricolor (GPS 180), Daedaleopsis sp. (Royan 8), Pholiota aurivella (GPS 142), Donkia pulcherrima (Neka 24D), Trametes sp. (GPS 130), Cyclocybe sp. (Darabkola 20), Ganoderma tsugae (GPS 186), and Hohenbuehelia auriscapium (GPS 196), respectively. Hohenbuehelia auriscalpium (GPS 196) and Donkia pulcherrima (Neka 24D) did not colonize grains over one month of incubation, while wild isolates such as Trametes sp. (GPS 130) and Ganoderma tsugae (GPS 186) filled spawn less than 15 days. Photographs were taken with a digital camera on the $30^{\text {th }}$ day of mycelial growth in the spawn. Each isolate was subjected to three independent replications. One of the replicates is shown in this figure.

\section{Discussion}

In this study, 40 Iranian wild-growing mushroom isolates (belonging to 21 different genera) were successfully adapted under solid media condition, including PDA and CEA media. However, few isolates failed to colonize grain-based or wood-based spawn. Forty isolates of mushrooms, eight wild mushroom isolates belonging to seven genera successfully produced fruiting bodies in wood-based lignocellulosic substrate in the small-scale cultivation tests. In general, domestication of several species of wild mushrooms have been reported globally, including Pleurotus giganteus (Klomklung et al. 2012), a range of wild tropical mushrooms (Thawthong et al. 2014), Panaeolus 
spp. (Bustillos et al. 2014), Agaricus flocculosipes and Agaricus subrufescens (Thongklang et al. 2014), Schizopyllum commune (Herawati et al. 2016), Flammulina velutipes (Rezaeian \& Pourianfar 2017), and Agaricus bisporus (Salmones et al. 2018).
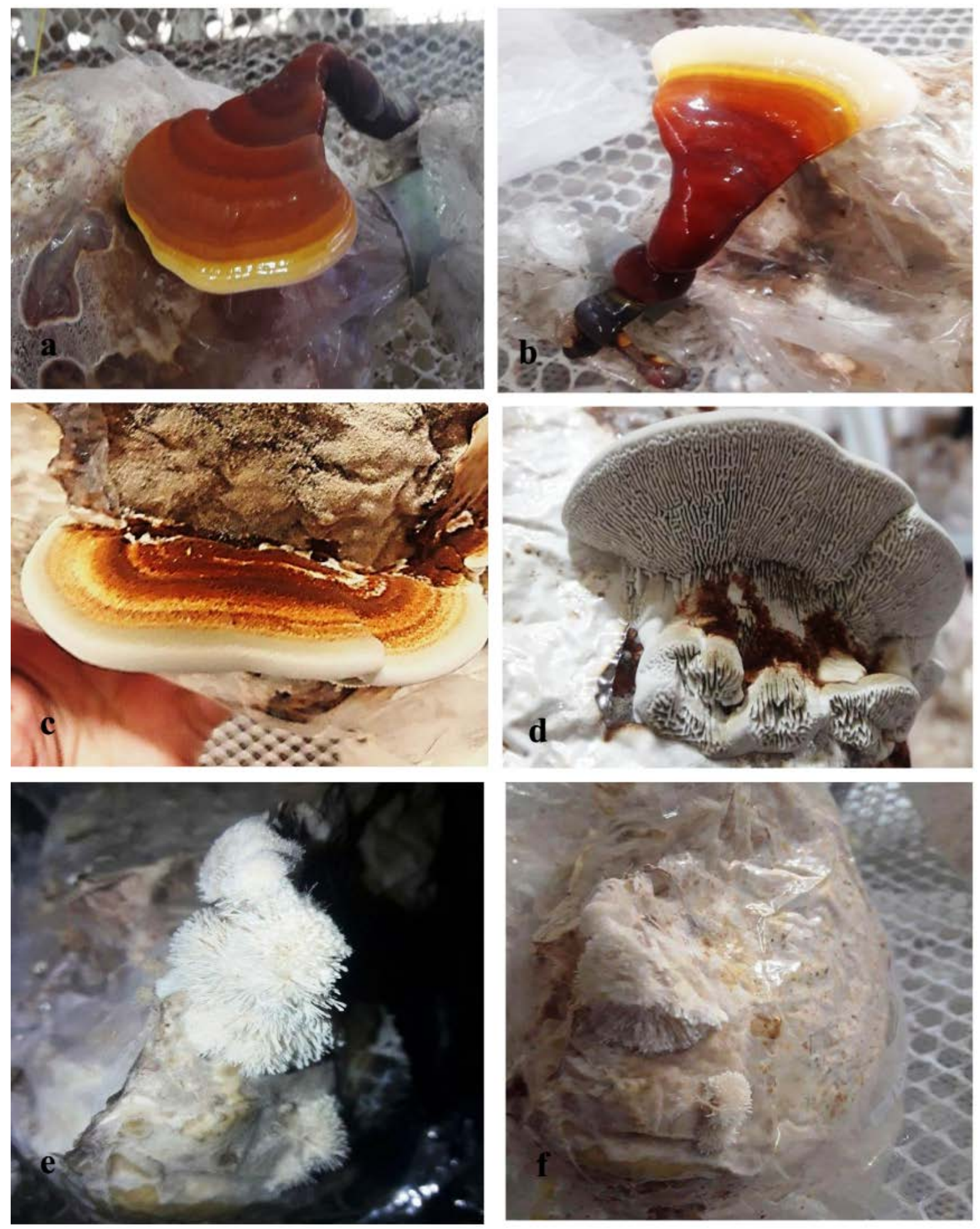

Fig. 6 - Successful fructification of wild mushroom isolates in wood chips-based substrate supplemented with wheat bran. Different developmental stages of Ganoderma tsugae (GPS 186), Daedaleopsis sp. (Royan 8), and Donkia pulcherrima (Neka 24D) are illustrated in panels a-b, c-d and e-f, respectively. Mature fruiting bodies of Ganoderma tsugae, Daedaleopsis sp., and Donkia pulcherrima appeared 90, 42, and 90 days, respectively, after transferring the fully colonized substrates to the cropping room. Each isolate was subjected to three independent replications. One of the replicates is shown in this figure. 

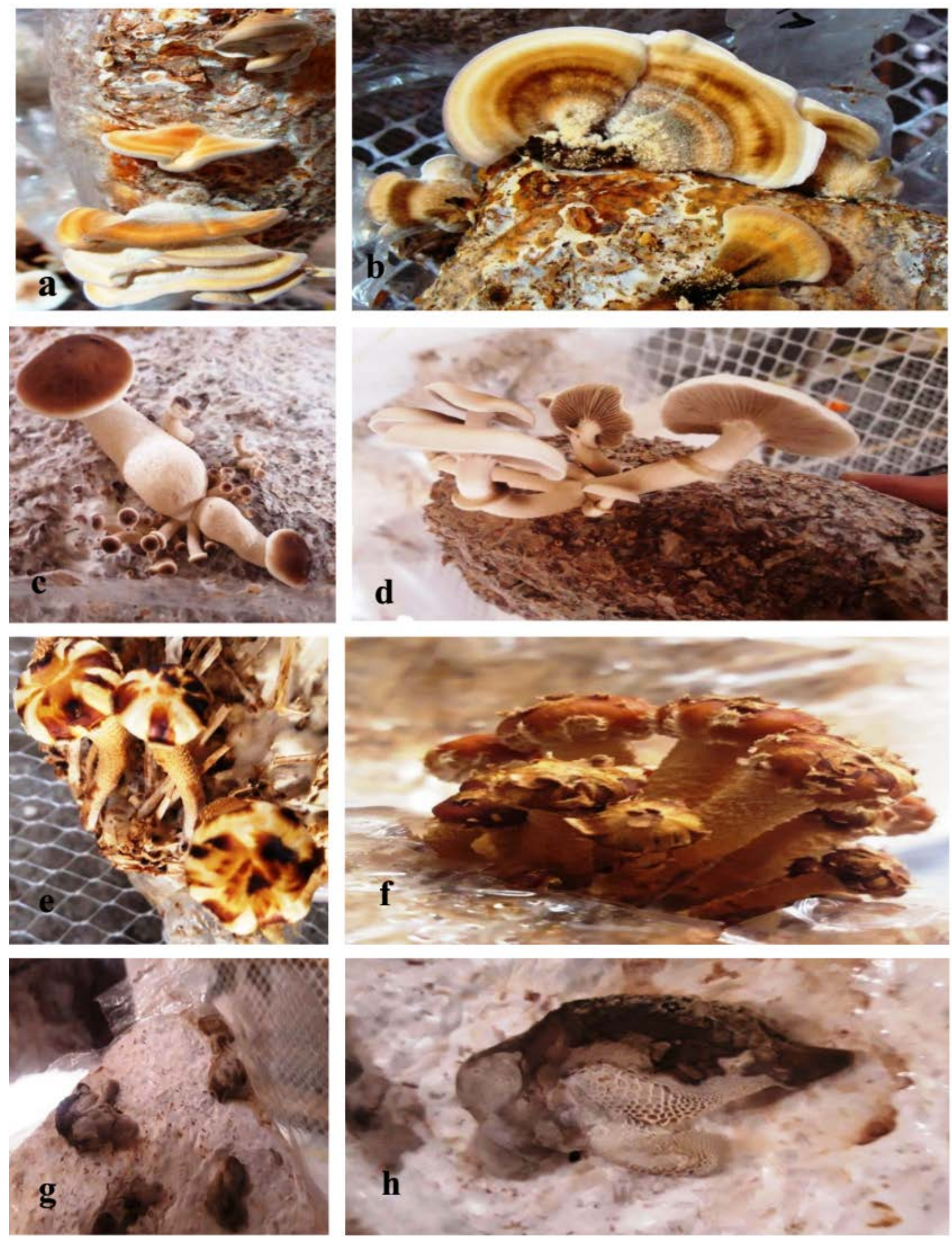

Fig. 7 - Successful fructification of wild mushroom isolates in wood chips-based substrate supplemented with wheat bran. Different developmental stages of Trametes sp. (strains of GPS 130 and Darabkola 13), Cyclocybe sp. (Darabkola 20), Pholiota aurivella (GPS 142), and Lenzites tricolor (GPS 180) are illustrated in panels a-b, c-d, e-f and g-h, respectively. Mature fruiting bodies of Trametes sp., Cyclocybe sp., Pholiota aurivella, and Lenzites tricolor appeared 58, 35, 50 , and 50 days, respectively, after transferring the fully colonized substrates to the cropping room. Photographs were taken with a digital camera during fructification. Each isolate was subjected to three independent replications. One of the replicates is shown in this figure. 
Cultivation of the Iranian wild mushrooms presented in this study is scarcely reported by researchers from the country. On the contrary, a number of studies from other countries have investigated cultivability of many of these wild species. Although there are taxonomic challenges with the genus Trametes and its related genera such as Lenzites and Daedaleopsis (Ueitele et al. 2018), the scientific knowledge of cultivation of many of the wild mushrooms presented in this study in East and Southeast Asia dates back to several decades ago (Thawthong et al. 2014, Luangharn et al. 2017). Similar to our findings, sawdust-based substrates enriched with wheat bran (or rice bran) have been frequently utilized to domesticate these wild mushrooms. In a relatively recent study, pinhead emergence of - Ganoderma neojaponicumin (from Malaysia) was found to be about two months (Tan et al. 2015), which was shorter than that of Ganoderma tsugae (3 months) domesticated in the current study. Similar results were also reported with Ganoderma austral (from Thailand) where development of pin-heads and maturation of fruiting bodies were completed in a short time (Luangharn et al. 2017). Several strains of wild G. lucidum was also collected and domesticated in Philippines with a period of about 17 days for fructification (from primordial formation to fruiting body maturation), even though no data was provided regarding the time required for pinhead emergence in the first harvest (Magday et al. 2014).

In the current study, Pholiota aurivella was also successfully cultivated in artificial substrates. Although no similar report is available regarding cultivability of Pholiota aurivella, domestication of another species, Pholiota adiposa, has been attempted in China (Rong et al. 2016). Similar to our findings, it took around 50 days for initiation of most of strains of Pholiota adiposa. However, one of the wild strains displayed better morphological traits and higher chemical contents as compared with the control strain. Cultivation of Pholiota adiposa and Pholiota microspora has been also reported from Japan (Huang \& Ohga 2017).

Fructification of Trametes versicolor collected from India was investigated using sawdust enriched with rice bran (Veena \& Pandey 2012). Primordial initiation was observed within 14-20 days of opening that was shorter than the period observed in our study. It took 30-45 days for the full development of the fruiting body that was very similar to the findings of the present study.

Cultivation of Agrocybe cylindracea in artificial substrate has been reported (Thawthong et al. 2014). In a recent study in Japan, this mushroom was successfully produced using $70 \%$ ceramic beads and 30\% of the nutrient solution sawdust (Huang \& Ohga 2017). However, it appears that no study has been undertaken to evaluate domestication of Donkia sp. and thus this study is the first to report successful cultivation of this wild mushroom.

The wild mushrooms adapted for cultivation in the present study have been also reported by other researchers in terms of their medicinal properties. Particularly, studies performed on the genera of Lenzites, Ganoderma and Trametes have revealed that these mushrooms had significant antimicrobial and anticancer properties (Vamanu \& Voica 2017, Yamac \& Bilgili 2006). Polysaccharides and sterols present in Ganoderma applanatum have shown beneficial effects for the treatment of diseases such as bronchitis, hepatitis, high blood pressure, types of tumors, and immune disorders (Kosanic et al. 2013). It was also found that the compounds extracted from the fruiting bodies of Ganoderma tsugae had high levels of phenolic acid, beta-carotene, lycopene, and vitamin C, all of which have high potential for scavenging free radicals (Mshandete 2014).

Species belonging to the genus Trametes (Turkey tail mushroom) have been shown to possess significant medicinal and therapeutic properties (Kamiyama et al. 2013). Orally administered preparations from the Trametes versicolor showed that up to 9 grams/day of a $T$. versicolor preparation is safe and tolerable in women with breast cancer in the postprimary treatment setting. This preparation may improve immune status in immunocompromised breast cancer patients following standard primary oncologic treatment (Torkelson et al. 2012). However, no final approval has been yet announced by the U.S. Food and Drug Administration (FDA). Phenolic and non-phenolic substances presented in this mushroom have also strong antioxidant properties (Mohd Nor Rasid 2010). Similarly, it has been reported that the compounds extracted from T. versicolor had a protective effect against the oxidation of red blood cells (Arteiro et al. 2012, Sun et al. 2014). Protein-polysaccharide complexes of this mushroom have anticancer properties and may boost the 
body immune system (Yang \& Zhang 2009, Jhan et al. 2016). Furthermore, T. hirsuta has been suited for its industrial uses (Vazirian et al. 2014).

The genus Lenzites is known as a rich source of antioxidant and antimicrobial compounds (Oyetayo et al. 2009). According to Abubakar et al. (2016), it was determined that the biological compounds presented in Lenzites quercina had antimicrobial effects.

Mushrooms of the genus Daedaleopsis belonging to Polyloraceae family are usually nonedible due to their hardness and woodiness (Shiono et al. 2005, Yu et al. 2008) Daedaleopsis confragosa was found to contain a large amount of phenolic compounds with antioxidant activity (Vidovic et al. 2011). In addition, Daedaleopsis tricolor has shown strong antifungal, antibacterial, and antioxidant properties (Zhao et al. 2013).

Pholiota adiposa has been reported to be rich in polysaccharides that have anti-tumor, antioxidant and antimicrobial properties (Dulger 2004, Deng et al. 2011). This mushroom is also rich in protein, essential amino acids, fiber, elements, vitamins, and carbohydrates. It was also determined that Pholiota aurivella contains lectin (Kawagishi et al. 1991). Lectins are well-known biologically active compounds that exhibit antimicrobial and anticancer activities (Abubakar et al. 2016).

Methanolic extracts from the fruiting bodies of Agrocybe (also known as Cyclocybe) aegerita contain significant amounts of various substances with high antioxidant properties (Petrovic et al. 2015). In a study performed by Chien et al. (2015), it was determined that Agrocybe cylindracea could significantly inhibit the growth of cancer cells.

\section{Conclusion}

The present study demonstrated the possibility of adaptation of several Iranian wild-growing medicinally important mushrooms to artificial locally available substrate. At the present, white button mushroom is still the most common cultivated mushroom in Iran (accounting for more than $90 \%$ of total mushroom production) followed by cultivated oyster mushrooms (Pleurotus spp.). The findings of this study with non-edible specialty mushrooms such as Ganoderma sp. or Trametes sp. might not be directly implicated in the mushroom industry. However, they may promote further pilot-scale and commercial-scale production of these medicinally important mushrooms. In addition, domestication of Donkia sp. was for the first time reported in this study, which may facilitate further investigations on its biological activities, including immune modulation and regulation, anticancer, antimicrobial, antioxidant, and probiotic properties. Further in-depth investigation would be warranted to evaluate different combinations of solid media, submerged media, and lignocellulosic agro-wastes in order to improve mycelial growth, biological efficiency, and other agronomic characteristics of the wild medicinal mushrooms.

\section{Acknowledgments}

This study was conducted as part of a research project financed by ACECR, Iran (code no 2283) granted to HR Pourianfar and MA Amoozegar.

\section{References}

Abubakar Z, Ogidi OC, Oyetayo VO. 2016 - Assessment of antistaphylococcal activity of ethanolic extract of Lenzites quercina (L) P. Karsten against clinical Staphylococcus species. Clinical Phytoscience 2, 8.

Arteiro JMS, Martins MR, Salvador, C, Candeias MF et al. 2012 - Protein-polysaccharides of Trametes versicolor: production and biological activities. Medicinal Chemistry Research 21, 937-943.

Asef MR. 2010 - Macrofungi flora of Arasbaran 4. Family Russulaceae. Rostaniha 12, 31-38.

Asef MR, Etemad V. 2016 - Identification of agaric fungi of Kheyroud Research Forest, Noshahr (Mazandaran province, N. Iran). Rostaniha 17, 19-27. 
Borhani A, Badalyan SM, Gharbyan NN, Mosazadeh SA. 2010 - Biodiversity and Distribution of Macronfungi Associated with Beeech Forest of Northern Iran (Case Study Mazandaran Province). World Application Science Journal 11, 151-158.

Bustillos RG, Dulay RMR, Kalaw SP, Reyes RG. 2014 - Optimization of culture conditions for mycelial growth and basidiocarp production of Philippine strains of Panaeolus antillarium and Panaeolus cyanescens. Mycosphere 5, 398-404.

Chien RC, Tasi SY, Lai EY, Mau JL. 2015 - Antiproliferative Activities of Hot Water Extracts from Culinary-Medicinal Mushrooms, Ganoderma tsugae and Agrocybe cylindracea (Higher Basidiomycetes) on Cancer Cells. International Journal of Medicinal Mushrooms 17, 453462.

Deng P, Zhang G, Zhou B, Lin R et al. 2011 - Extraction and in vitro antioxidant activity of intracellular polysaccharide by Pholiota adiposa SX-02. Journal of Bioscience and Bioengineering 111, 50-54.

Dulger B. 2004 - Antimicrobial activity of the macrofungus Pholiota adiposa. Fitoterapia 75, 95397.

Erkel EI. 2009 - The effect of different substrate mediums on yield of Ganoderma locium (Fr.) Karst. Journal of Food, Agriculture and Environment 7, 841-844.

Ershad D. 2009 - Fungi of Iran. $3^{\text {rd }}$ edition. Agricultural Research, Education and Extension Organization, Publication No 10, Tehran, Iran.

Ghanbari J, Pourianfar HR, Sarmad Nabavi M, Mostafavi Mendi E. 2015 - A comparative study of agronomic characteristics in several Iranian wild isolates of the button mushroom, Agaricus bisporus. International Journal of Advanced Research 3, 230-235.

Herawati E, Arung ET, Amirta R. 2016 - Domestication and Nutrient Analysis of Schizopyllum commune, Alternative Natural Food Sources in East Kalimantan. Agriculture and Agricultural Science Procedia 9, 291-296.

Huang P, Ohga S. 2017 - Utilization of ceramic beads for edible mushrooms cultivation. Advances in Microbiology 7, 853-862.

Jhan MH, Yeh CH, Tsai CC, Kao CT et al. 2016 - Enhancing the Antioxidant Ability of Trametes versicolor Polysaccharopeptides by an Enzymatic Hydrolysis Process. Molecules. 21.

Kamalebo HM, Malale HNSW, Ndabaga CM, Degreef J, De Kesel A. 2018 - Uses and importance of wild fungi: traditional knowledge from the Tshopo province in the Democratic Republic of the Congo. Journal of Ethnobiology and Ethnomedicine 14, 13.

Kamiyama M, Horiuchi M, Umano K, Kondo K et al. 2013 - Antioxidant/anti-inflammatory activities and chemical composition of extracts from the mushroom Trametes versicolor. International Journal of Nutrition and Food Sciences 2, 85-91.

Kawagishi H, Abe Y, Nagata T, Kimura A, Chiba S. 1991 - A lectin from the Mushroom Pholiota aurivella. Agricultural and Biological Chemistry 55, 2485-2489.

Keypour S, Riahi H, Borhani A, Shayan MRA, Safaie N. 2014 - Survey on wood decay fungi Ganoderma species (Ganodermataceae; Polyporales) from Guilan and Mazandaran, Iran. International Journal of Agriculture and Biosciences 3, 132-135.

Klomklung N, Karunarathna SC, Chukeatirote E, Hyde KD. 2012 - Domestication of wild strain of Pleurotus giganteus. Sydowia 64, 39-53.

Kosanic M, Rankovic B, Dasic M. 2013 - Antioxidant and antimicrobial properties of mushrooms. Bulgarian Journal of Agricultural Science 19, 1040-1046.

Lindequist U, Niedermeyer TH, Jülich WD. 2005 - The pharmacological potential of mushrooms. Journal of Evidence-Based Integrative Medicine 2, 285-299.

Luangharn T, Karunarathna SC, Khan S, Xu JC et al. 2017 - Antibacterial activity, optimal culture conditions and cultivation of the medicinal Ganoderma australe, new to Thailand. Mycosphere 8, 1108-1123.

Magday JJ, Dulay RM, Bungihan ME. 2014 - Optimization of mycelial growth and cultivation of fruiting body of Philippine wild strain of Ganoderma lucidum. Current Research in Environmental \& Applied Mycology, 4, 162-172. 
Masoumi F, Pourianfar HR, Masoumi A, Mostafavi Mendi E. 2015 - A study of mycelium characterization of several wild genotypes of the button mushroom from Iran. International Journal of Advanced Research 3, 236-246.

Miles PG, Chang ST. 2004 - Mushrooms: Cultivation, Nutritional Value, Medicinal Effect, and Environmental Impact. CRC Press.

Mohd Nor Rasid N. 2010 - Diversity And Antioxidant Activity Of Trametes Fr. In Malaysia. Faculty of Science University Malaya Kuala Lumpur.

Mshandete AM. 2014 - Cytotoxicity and Antioxidant Activities of Ganoderma tsugae - A Basidiomycetes Mushroom Indigenous from Tanzania. International Journal of Life Sciences 3, 189-197.

Oyetayo OV. 2011 - Medicinal uses of mushrooms in Nigeria: towards full and sustainable exploitation. African Journal of Traditional, Complementary and Alternative Medicines 8, 267-274.

Oyetayo OV, Dong CH, Yao YJ. 2009 - Antioxidant and Antimicrobial Properties of Aqueous Extract from Dictyophora indusiata. The Open Mycology Journal 3, 20-26.

Petrovic J, Glamoclija J, Dejan S, Ciric A et al. 2015 - Nutritional value, chemical composition, antioxidant activity and enrichment of cream cheese with chestnut mushroom Agrocybe aegerita (Brig.) Sing. Journal of Food Science and Technology 52, 6711-6718.

Rahnama K, Habibi R. 2015 - First report of Neurospora on Corylus avellana in natural forest of Iran. Journal of Yeast and Fungal Research 6, 31-36.

Rezaeian SH, Pourianfar HR. 2017 - A Comparative Study on Bioconversion of Different Agro Wastes by Wild and Cultivated Strains of Flammulina velutipes. Waste and Biomass Valorization 8, 2631-2642.

Rong CB, Song S, Niu YR, Xu F et al. 2016 - Selection of a highly productive strain of Pholiota adiposa. Mycosphere 7, 226-235.

Royse DJ, Baars J, Tan Q. 2017 - Current overview of mushroom production in the world. In: Cunha Zied D, Pardo-Giménez A (eds). Edible and Medicinal Mushrooms: Technology and Applications, 1st Ed. Wiley-Blackwell, Chapter 2.

Salmones D, Gaitan-Hernandez R, Mata G. 2018 - Cultivation of Mexican wild strains of Agaricus bisporus, the button mushroom, under different growth conditions in vitro and determination of their productivity. Biotechnologie, Agronomie, Société et Environnement 22, 45-53.

Semwal KC, Stephenson SL, Bhatt VK, Bhatt RP. 2014 - Edible mushrooms of the Northwestern Himalaya, India: a study of indigenous knowledge, distribution and diversity. Mycosphere 5, 440-461.

Shiono Y, Tamesada Y, Muravayev YD, Murayama T, Ikeda M. 2005 - NPhenethylhexadecanamide from edible mushroom Laetiporus Sulphareus. Natural Product Research 19, 363-366.

Sun X, Sun Y, Zhang Q, Zhang H et al. 2014 - Screening and comparison of antioxidant activities of polysaccharides from Coriolus versicolor. International Journal of Biological Macromolecules 69, 12-19.

Tan WC, Kuppusamy UR, Phan CW, Tan YS et al. 2015 - Ganoderma neo-japonicum Imazeki revisited: Domestication study and antioxidant properties of its basidiocarps and mycelia. Scientific Reports 5, p. 12515.

Thawthong A, Karunarathna SC, Thongklang N, Chukeatirote E et al. 2014 - Discovering and domesticating wild tropical cultivatable mushrooms. Chiang Mai Journal of Science 41, 731764.

Thongklang N, Sysouphanthong P, Callac P, Hyde KD. 2014 - First cultivation of Agaricus flocculosipes and a novel Thai strain of A. subrufescens. Mycosphere 5, 814-820.

Torkelson CJ, Sweet E, Martzen MR, Sasagawa M et al. 2012 - Phase 1 clinical trial of Trametes versicolor in women with breast cancer. ISRN oncology 2012.

Ueitele ISE, Chimwamurombe PM, Kadhila NP. 2018 - Molecular Phylogeny of Trametes and Related Genera from Northern Namibia. Jordan Journal of Biological Sciences 11, 99-105. 
Vamanu E, Voica A. 2017 - Total phenolic analysis, antimicrobial and antioxidant activity of some mushroom tinctures from medicinal and edible species, by in vitro and in vivo tests. Scientific Bulletin. Series F. Biotechnologies 21, 318-324.

Vazirian M, Dianat S, Manayi A, Ziari R et al. 2014 - Anti-inflammatory effect, total polysaccharide, total phenolics content and antioxidant activity of the aqueous extract of three basidiomycetes. Research Journal of Pharmacognosy 1, 15-21.

Veena SS, Pandey M. 2012 - Physiological and cultivation requirements of Trametes versicolor, a medicinal mushroom to diversify Indian mushroom industry. Indian Journal of Agricultural Sciences 82, p.672.

Vidovic S, Zekovic Z, Mujic I, Lepojevic Z et al. 2011 - The antioxidant properties of polypore mushroom Daedaleopsis confragosa. Central European Journal of Biology 6, 575-582.

Wachtel-Galor S, Yuen J, Buswell JA, Benzie IFF. 2011 - Ganoderma lucidum (Lingzhi or Reishi): A Medicinal Mushroom. In: Benzie IFF, Wachtel-Galor S. editors. Herbal Medicine: Biomolecular and Clinical Aspects. 2nd edition. Boca Raton (FL): CRC Press.

Yamac M, Bilgili F. 2006 - Antimicrobial activities of fruit bodies and/or mycelial cultures of some mushroom isolates. Pharmaceutical Biology 44, 660-667.

Yang L, Zhang LM. 2009 - Chemical structural and chain conformational characterization of some bioactive polysaccharides isolated from natural sources. Carbohydrate Polymers 76, 349-361.

Yu L, Zhang B, Geza R, Szalvay K et al. 2008 - Protein HGF1 from edible mushroom Grifola frondosa is a novel 8kda class I hydrophobin that forms rodlets in compressed monolayers. Microbiology 154, 1677-1685.

Zhao JY, Feng T, Li ZH, Dong ZJ et al. 2013 - Sesquiterpenoids and an ergosterol from cultures of the fungus Daedaleopsis tricolor. Natural Products and Bioprospecting 3, 271-276. 